University of Nebraska - Lincoln

DigitalCommons@University of Nebraska - Lincoln

Faculty Publications, Department of Child, Youth, and Family Studies

Child, Youth, and Family Studies, Department of

2017

Implementing the Academy of Nutrition and Dietetics

Benchmarks for Nutrition Education for Children: Child-Care

Providers' Perspectives

Dipti A. Dev

University of Nebraska-Lincoln, ddev2@unl.edu

Virginia Carraway-Stage

East Carolina University, carrawaystagev@ecu.edu

Daniel J. Schober

DePaul University, dschober@depaul.edu

Brent A. McBride

University of Illinois at Urbana-Champaign, brentmcb@illinois.edu

Car Mun Kok

University of Nebraska-Lincoln, carmun@huskers.unl.edu

Follow this and additional works at: https://digitalcommons.unl.edu/famconfacpub See next page for additional authors

Part of the Developmental Psychology Commons, Dietetics and Clinical Nutrition Commons, Family,

Life Course, and Society Commons, Other Psychology Commons, and the Other Sociology Commons

Dev, Dipti A.; Carraway-Stage, Virginia; Schober, Daniel J.; McBride, Brent A.; Mun Kok, Car; and Ramsay, Samantha, "Implementing the Academy of Nutrition and Dietetics Benchmarks for Nutrition Education for Children: Child-Care Providers' Perspectives" (2017). Faculty Publications, Department of Child, Youth, and Family Studies. 151.

https://digitalcommons.unl.edu/famconfacpub/151

This Article is brought to you for free and open access by the Child, Youth, and Family Studies, Department of at DigitalCommons@University of Nebraska - Lincoln. It has been accepted for inclusion in Faculty Publications, Department of Child, Youth, and Family Studies by an authorized administrator of DigitalCommons@University of Nebraska - Lincoln. 


\section{Authors}

Dipti A. Dev, Virginia Carraway-Stage, Daniel J. Schober, Brent A. McBride, Car Mun Kok, and Samantha Ramsay 


\title{
Implementing the Academy of Nutrition and Dietetics Benchmarks for Nutrition Education for Children: Child-Care Providers' Perspectives
}

\author{
Dipti A. Dev, PhD; Virginia Carraway-Stage, PhD, RDN, LDN; Daniel J. Schober, PhD, MPH; \\ Brent A. McBride, PhD; Car Mun Kok, PhD; Samantha Ramsay, PhD, RD
}

\begin{abstract}
Background National childhood obesity prevention policies recommend that childcare providers educate young children about nutrition to improve their nutrition knowledge and eating habits. Yet, the provision of nutrition education (NE) to children in child-care settings is limited.

Objective - Using the 2011 Academy of Nutrition and Dietetics benchmarks for NE in child care as a guiding framework, researchers assessed childcare providers' perspectives regarding delivery of NE through books, posters, mealtime conversations, hands-on learning, and sensory exploration of foods to young children (aged 2 to 5 years).

Design - Using a qualitative design (realist method), individual, semistructured interviews were conducted until saturation was reached.

Participants/setting - The study was conducted during 2012-2013 and used purposive sampling to select providers. Final sample included 18 providers employed full-time in Head Start or state-licensed center-based child-care programs in Central Illinois.

Main outcome measure - Child-care providers' perspectives regarding implementation of NE.

Statistical analyses performed - Thematic analysis to derive themes using NVivo software.

Results - Three overarching themes emerged, including providers' motivators, barriers, and facilitators for delivering NE to children. Motivators for delivering NE included that NE encourages children to try new foods, NE improves children's knowledge of healthy and unhealthy foods, and NE is consistent with children's tendency for exploration. Barriers for delivering NE included that limited funding and resources for hands-on experiences and restrictive policies. Facilitators for delivering NE included providers obtain access to feasible, low-cost resources and community partners, providers work around restrictive policies to accommodate $\mathrm{NE}$, and mealtime conversations are a feasible avenue to deliver NE. Providers integrated mealtime conversations with NE concepts such as food-based sensory exploration and health benefits of foods.

Conclusions - Present study findings offer insights regarding providers' perspectives on implementing NE in child care. Drawing from these perspectives, registered dietitian nutritionists can train providers about the importance of NE for encouraging healthy eating in children, integrating NE with mealtime conversations, and practicing low-cost, hands-on NE activities that meet the food safety standards for state licensing. Such strategies may improve providers' ability to deliver NE in child-care settings.
\end{abstract}

Keywords: Child-care providers, Nutrition education, Head Start program, Child and Adult Care Food Program, Childhood obesity prevention

C hild-care settings offer an ideal environment for implementing nutrition education (NE) to positively influence preschool-aged children's (aged 2 to 5 years) dietary intake and reach large segments of low-income, minority children and their families at a higher risk for obesity. ${ }^{1-4}$ In support of this research regarding the importance of NE for improving children's dietary intake, a Position Paper of the Academy of Nutrition and Dietetics (Academy) proposed benchmarks for nutrition in child-care settings. ${ }^{2}$ The benchmarks provide child-care providers with guidance for meeting children's nutrition needs and promote optimal growth and development. ${ }^{2}$ Specifically, regarding benchmarks for NE, the Academy recommends that NE for children should be a component of the child-care program where child-care providers should use material resources (ie, books and posters), mealtime conversations, hands-on learning, and sensory exploration of foods for NE in early childhood classroom settings. ${ }^{2}$ These resources are commonly cited components of NE interventions $s^{5-8}$ and have been

D. A. Dev is an assistant professor, Department of Child, Youth, and Family Studies, University of Nebraska, Lincoln; at the time of the study, she was a doctoral degree student, Division of Nutritional Sciences, University of Illinois at Urbana-Champaign, Urbana.

V. Carraway-Stage is an assistant professor, Department of Nutrition Science, East Carolina University, Greenville.

D. J. Schober is a clinical assistant professor, DePaul University, Chicago, IL.

B. A. McBride is a professor, Department of Human and Community Development, University of Illinois at Urbana- Champaign, Urbana.

C. M. Kok is a 4-H Youth Development Program advisor, University of California Cooperative Extension, Lakeport; at the time of the study, she was a doctoral degree student, Department of Child, Youth, and Family Studies, University of Nebraska, Lincoln.

S. Ramsay is an assistant professor, School of Family and Consumer Sciences, University of Idaho, Moscow.

Corresponding author - Dipti A. Dev, PhD, Department of Child, Youth, and Family Studies, 135 Home Economics Bldg, Lincoln, NE 68588- 0236; email ddev2@unl.edu 
shown to increase preschoolers' ability to distinguish between healthy and unhealthy foods, ${ }^{9}$ improve preferences for new and healthier foods, ${ }^{10,11}$ and increase fruit and vegetable consumption. ${ }^{5,10}$ More importantly, when children establish these positive dietary behavior changes, they can carry those improved health behaviors into home environments. ${ }^{12}$

Unfortunately, the provision of the Academy's NE benchmarks in child care is a challenge because recent quantitative reports recognize that many child-care providers may not deliver NE. ${ }^{13}$ Studies in Oklahoma, ${ }^{14}$ Minnesota, Wisconsin, ${ }^{15}$ and North Carolina ${ }^{16}$ indicated $<25 \%$ of child-care providers offered NE to children. Regarding the Academy's benchmark on delivering NE through mealtime conversations, $<40 \%$ of providers talked to children about healthy foods ${ }^{16}$ and meaningful mealtime nutrition conversations were rare. ${ }^{16,17}$ Similarly, nutritionrelated posters, pictures, or books were rarely seen in child-care facilities. ${ }^{17}$ Further, a study in Rhode Island reported that $90 \%$ of providers disagreed or strongly disagreed that the children in their care were exposed to nutrition-related books, cooking, and other hands-on food activities. ${ }^{18}$ Findings from these quantitative studies underscore the need for a qualitative study to better understand child-care provider perspectives regarding implementing the Academy's NE benchmarks for children in their care.

The implementation of the Academy's NE benchmarks depends on child-care providers who are accountable for delivering NE to more than 1 million children annually. ${ }^{2,3}$ Providers' ability to teach nutrition is associated with improvement in their perspectives (ie, knowledge and clarifying misconceptions) and is a priority for childhood obesity prevention. ${ }^{19}$ These findings suggest that understanding child-care providers' perspectives is an essential component of NE promotion. ${ }^{19}$ Limited research has focused on understanding child-care providers' perspectives regarding incorporating NE in their classroom. A recent qualitative study reported providers' lack of knowledge regarding their role in teaching nutrition and incorporating developmentally appropriate practices for NE in child-care settings. ${ }^{19}$ Providers also lacked an understanding of what NE entails, its definition, and educational guidelines. ${ }^{20,21}$ The present study aims to bridge this disconnect by using the Academy's benchmarks as a guiding framework, to define NE and gain a better understanding of providers' perspectives regarding implementing specific NE benchmarks in their classroom.

Therefore, the purpose of the present qualitative study was to explore child-care providers' perspectives regarding delivering NE to young children through books, posters, mealtime conversations, hands-on learning, and sensory exploration of foods as recommended by the Academy's benchmarks.

\section{Methods}

\section{Research Design}

To explore providers' perspectives regarding NE, researchers conducted in-depth, face-to-face, individual semistructured qualitative interviews with child-care providers. Data were analyzed using thematic analysis. ${ }^{22}$ Researchers with expertise in nutrition, child development, public health, child care, and qualitative methods designed and executed the study. The University of Illinois at Urbana-Champaign Institutional Review Board approved the study methods.

\section{Sampling and Recruitment}

During 2012, 118 providers from 24 licensed child-care centers in Central Illinois completed a survey as part of a quantitative study on their mealtime feeding practices. ${ }^{13}$ Of these 118 child-care providers who participated in the primary quantitative study, 90 signed a written informed consent to participate in an interview (secondary qualitative study) in the case that they were contacted. All 90 providers were fulltime child-care workers, had direct contact with preschoolers (aged 2 to 5 years), and were responsible for supervising meals or snacks. ${ }^{13}$ From a sampling frame of 90 providers, potential participants were selected for the secondary qualitative study by using purposive sampling to obtain a balanced perspective regarding the implementation of the Academy's benchmarks. ${ }^{23}$ Providers were sampled based on the varying childcare contexts (Head Start, Child and Adult Care Food Program [CACFP] efunded, or non-CACFP), ${ }^{24}$ age, education, and years of experience, a sampling approach also used in previous qualitative studies. ${ }^{25,26}$ This approach ensured diversity of child-care providers was captured from the primary quantitative study sample. All providers who were contacted agreed to participate in an interview. Participants received a $\$ 25$ gift card for participating.

\section{Measurement Instrument}

The lead author interviewed all providers using a semistructured interview guide from the About Feeding Children Study ${ }^{23,27}$ to examine providers' perceptions regarding 18 Academy benchmarks related to feeding practices such as role modeling, serving meals family style, fostering selfregulation in eating, avoiding controlling feeding practices, communicating with parents regarding their child's nutrition, and offering NE. ${ }^{2}$ The detailed methodology for this qualitative study has been published previously, ${ }^{28}$ in a study in which child-care providers' perceptions regarding familystyle meal service (where children select their own portions and serve themselves) were examined. This article specifically focuses on exploring child-care providers' perceptions regarding implementation of the Academy's NE benchmark, NE for children should be a component of the child-care program through inclusion of two key actions: providers incorporate NE into their daily routines with children through books, posters, hands-on experiences, and mealtime conversations; and providers help children learn about food by engaging their senses and touching and smelling new foods is encouraged as a step toward tasting the food. ${ }^{2}$ Providers' perspectives regarding the Academy's benchmarks were gathered through the closed card sorting method in which participants sort a series of cards, each labeled with specific content, into the defined groups. ${ }^{27,29}$ Providers were presented with a stack of 18 cards that listed the Academy' benchmarks and were asked to sort the cards into three piles-one pile for the benchmarks that their child-care center uses, one for those the center does not use, and one for those that have not heard of or are unsure about. For the pile of cards that the center uses, providers were asked to sort the cards again into three piles: those benchmarks they find are easy to do, they sometimes find hard to do, and find really hard to do; and then follow-up questions were asked regarding why it was easy, sometimes hard, or very hard to deliver NE to better understand childcare providers' perceptions regarding delivering NE. For the 
detailed interview protocol see the Figure (available online at www. jandonline.org). Before data collection, an interdisciplinary team of researchers reviewed the interview protocol and the lead author (also the interviewer) completed training on strategies to remain open, unbiased, and nonjudgmental during the interview. ${ }^{30}$ The lead author pilot-tested the interview protocol for face validity with seven child-care providers, and received observer feedback to guide revisions for the interview protocol. 31,32 The interview protocol was modified to focus on providers' perceptions regarding the Academy's benchmarks and exclude questions about mealtime environment and roles. The protocol was modified to maintain the interview duration between 45 minutes and 1 hour to reduce participant burden.

\section{Data Collection}

The lead author, who had no prior relationship with the childcare centers or providers, conducted one-on-one, face-to-face interviews with 15 child-care providers until data saturation was reached or no new relevant information was revealed. ${ }^{32}$ An additional three interviews confirmed the findings, with no new information revealed, bringing the final sample to 18 child-care providers. Interviews were conducted between August and November 2012 at the participant's child-care setting, and lasted approximately 45 to 60 minutes. To encourage the participants to speak freely, all interviews were completed in an unoccupied room. ${ }^{30}$ Before the interview, the lead author explained to the provider the reasons for doing the study, assured that answers would not be shared with anyone outside the study team, and provided the opportunity to ask questions before the interview. Interviews were audiorecorded and the participant's identity was not mentioned during the recording. ${ }^{28}$ Immediately after the interview, the interviewer prepared field notes to summarize the participant's responses from the interview. All provider responses were de-identified and pseudonyms were used for each provider during data analysis and for writing the results.

\section{Data Analysis}

All interviews were transcribed verbatim by a professional transcription agency and imported into NVivo 9 for analysis. ${ }^{33} \mathrm{~A}$ thematic approach was used where the data were coded using six steps of thematic analysis, ${ }^{22}$ which refers to identifying, analyzing, and reporting patterns or themes across the entire data set. ${ }^{22}$ In this study, thematic analysis was grounded in the epistemologic assumptions of the realist method, which reports participants' reality, meanings, and experiences. ${ }^{22}$

Two authors (including the lead author) independently read each transcript twice and identified a set of codes, code definitions, and themes. These coders then met to achieve consensus about codes and themes through verbal agreement. Decision for agreement was yes or no; when disagreement occurred, the two coders modified and refined the coding and themes until any disagreements were resolved. ${ }^{21,34}$ Both coders agreed with codes and themes. The coders discussed any disagreement mainly regarding naming the themes and achieved consensus through verbal agreement. Authors who did not code the transcripts verified that the themes were supported by the codes and quotations. Throughout the data collection and analysis process, the lead author monitored researchers' biases to ensure that results (codes and themes) were accurately represented based on participant responses, through ongoing peer debriefing consultations and frequent research team meetings. ${ }^{30}$

\section{Results}

The final sample included 18 child-care providers who shared their perspectives regarding implementing the Academy's NE benchmarks (see the Table). All providers $(n=18)$ were nonHispanic black $(n=9)$ and non-Hispanic white $(n=9)$ women. All 18 providers were employed full-time and represented 14 unique child-care settings. On average, the providers were aged 42 years and had 12 years of experience as a child-care provider. Just more than half of providers had some college or technical school or less education.

For card sort results, all providers reported that they incorporated NE into daily routines through books, hands-on experiences, and mealtime conversations. From the 18 providers, 9 providers reported that this practice was easy to implement, 7 reported it was sometimes hard, and 1 reported it was very hard. Further, among 17 providers who reported that they helped children learn about food by engaging their senses, 8 providers reported that this was easy to implement, 7 reported it was sometimes hard, and 2 providers reported it was very hard. One provider reported that they did not deliver NE through engaging children's senses.

Based on the card sorting, providers responded to followup questions regarding the reason for their responses; that is, why it is easy, hard, or very hard to implement the NE benchmark. Three overarching themes emerged regarding providers' perspectives for implementing the Academy's NE benchmarks for young children attending child care: motivators for delivering $\mathrm{NE}$, barriers to delivering $\mathrm{NE}$, and facilitators for delivering $\mathrm{NE}$ to children attending child care.

\section{Motivators}

Three themes emerged from the data regarding motivators or reasons providers gave for delivering NE to children attending child care. Child-care providers were motivated to deliver NE to children because NE encourages children to try new foods, NE improves children's knowledge of healthy and unhealthy foods, and NE is consistent with children's tendency for exploration. Each theme is described below:

Motivator 1: Providers Perceived NE Encourages Children to Try New Foods. Providers reported delivering NE because it encouraged children to try new foods. Trisha explained integrating NE (engaging senses) during mealtime conversations:

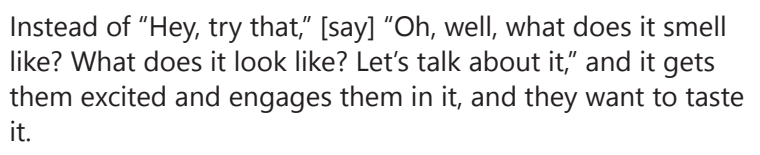

When asked why she engages children in food-based sensory exploration, Erin responded:

Because if they smell it, they like it, they're going to probably try it ... when they taste it, they're going to eat it. 
Table. Demographic characteristics of a cohort of 18 childcare providers in Central Illinois participating in semistructured interview data collection on their implementation of the Academy of Nutrition and Dietetics' nutrition education benchmarks ${ }^{2}$ with children aged 2 to 5 years

\begin{tabular}{lc}
\hline Characteristic & Result \\
\hline Affiliation & $\mathrm{n}$ \\
Head Start & 6 \\
Child and Adult Care Food Program & 6 \\
Non-Child and Adult Care Food Program & 6 \\
Race/ethnicity & 9 \\
Non-Hispanic black & 9 \\
Non-Hispanic white & \\
Education & 10 \\
Some college or technical school & 8 \\
College graduate &
\end{tabular}

mean \pm standard deviation

Age (y)

$41.52 \pm 13.2$

Experience as child-care teacher $(\mathrm{y})$

$11.7 \pm 9.1$

Teachers incorporate nutrition education into their daily routines with children, through books, posters, hands-on experiences, and mealtime conversations

Practiced, easy

Practiced, sometimes hard

Practiced, very hard

Did not practice

Teachers help children learn about food by engaging their senses, such as smell, touch, and taste, where touching and smelling new foods is encouraged as a step toward tasting

Practiced, easy

Practiced, sometimes hard

Practiced, very hard

Did not practice
Motivator 2: Providers Perceived NE Improves Children's Knowledge of Healthy and Unhealthy Foods. In addition to mealtime conversations, providers discussed using posters and hands-on activities as important to improving children's knowledge of healthy and unhealthy foods. Megan stated,

We show children the food plate that has the portions and the meat, grain, protein, and.and I feel like that's good to teach them that so they know what's healthy.

Michelle explained an activity regarding organizing healthy and unhealthy foods in the Food Pyramid that increased children's curiosity for understanding the concept of such foods. She explained:

They [children] ask all the time, because they get it in their head, healthy, unhealthy, healthy .so they're constantly, is this healthy?....This is unhealthy, isn't it? So, I mean, they're 3 and 4 years old. They understand.

Motivator 3: Providers Perceived NE Is Consistent with Children's Tendency for Exploration. Providers frequently discussed taking advantage of children's natural tendency toward curiosity and sensory exploration as opportunity for hands-on classroom-based NE. Ashley stated:

It [engaging children's senses] is easy to do because that's what kids do anyway. They want to touch it and they want to smell it, and they want to taste it. So, it's very easy for kids to do that.

Another provider, Marisa, held a similar view-specifically in terms of children's desire to learn through the sense of touch:

It [engaging children's senses] is easy to do because a 2-year-old puts her hands in everything, and that is the next step to tasting, become familiar with it. So that's something they naturally do.

Becky expressed that food-based sensory exploration is universal throughout her classroom:

Children get the feel of different textures of food and different tastes in food, and we try to encourage that in everything we do in our classroom. Smell it, touch it, feel it.

Taken together, these three motivators for implementing NE suggest that providers perceived it was important to deliver NE to children to encourage children's healthy eating.

Barriers

Two themes emerged from the data regarding barriers or difficulties child-care providers faced for delivering NE to children in their care. Each theme is described below:

Barrier 1: Providers Perceived They Have Limited Funding and Resources for Delivering NE. Providers expressed their barriers for implementing hands-on activities owing to a lack of monetary resources. Maureen expressed a need for "a master list of resources of things that are free, available at a small cost, and things that are available for accredited centers." Trisha explained: "The only thing that would be hard would be like the books and
You drink your milk, it helps you build strong bones, it builds your muscles. Every time she drinks her milk now, she says, "I'm gonna drink my milk because it's healthy for me, because it makes me strong." 
posters because we don't have access to it here." In addition, lack of funding to support NE education through hands-on activities was a common concern. Megan remarked:

\section{I think that's one of the only things that hinders us in doing that [activities] because we'll want to get a bunch of different kinds of things and have kids look at them, smell them, and eat them. But sometimes there's not money for that.}

Megan also mentioned an example of how management denied certain food activities because of insufficient funds:

Well, you already did one food activity this month, you only get $\$ 5$ and you already used $\$ 3 . .$. so we can't get you everything you need.

Barrier 2: Providers' Perceived Policies Restrict Certain Aspects of NE. Providers reported multilevel policies as a common barrier to NE. Megan described how center-level policies create challenges to sensory-based, hands-on NE:

Sometimes we have a problem getting things.like food and things because we can't have anything that's brought in from somebody else's home. It has to be from the store.

Maureen described uncertainty reconciling NE recommendations from accrediting organizations with mandates from state policies:
Accreditation will say, "Do cooking projects for children. Let them crack eggs. Let them measure. Let them spill. Let them mix these ingredients." Then, you go to [Department of Children and Family Services] and public health and they say, "You may not use raw eggs." So it's the two do not go together.

\section{Facilitators}

Three themes emerged from the data regarding facilitators or factors that promoted child-care providers' ability to deliver NE to children. Each theme is described below.

Facilitator 1: Providers Obtain Access to Feasible, Low-Cost Resources and Community Partners to Facilitate Implementation of NE. Providers who delivered NE to children perceived that NE through books, posters, and mealtime conversations was easy to implement owing to their feasibility. These approaches to NE are easy to access and deliver, reduce lesson planning time, and offer repeated visual cues to educate children about nutrition.

Fiona explained:

Because we have it right in front of us. We have books in our dramatic play areas, and it's actually information that we can read over and over.

Posters and books were easy to use when providers could easily access these materials through program support and community partners. Megan explained,

I would give a lot of credit to the program and the resources that we find or that find us. We have a lot of people that cooperate with us, that donate materials. Our program is really good for working with other places, and.it gives us. a lot of chances to be able to have the materials that we need.

Jade mentioned "free websites and the book club and library" as good resources as well. Providers suggested two primary approaches to access resources for hands-on NE. First, providers suggested engaging parents in NE, such as asking parents to bring in fruit for hands-on learning. Second, providers suggested using existing community partners (eg, Cooperative Extension) to support NE.

Facilitator 2: Providers Accommodate NE by Working Around Policies That Restrict Certain Aspects of NE. Providers who implemented hands-on NE cooking activities with children used various tactics to work around regulations that may be limiting in some areas. Dana suggested,

We don't have stoves and those things, but we make modeling clay, home-made ice-cream, fruit-they learn how to cut fruit so we consider that a cooking project. ...There are cooking projects that you can do that are considered cooking without actually cooking the food.

Another provider, Maureen, explained:

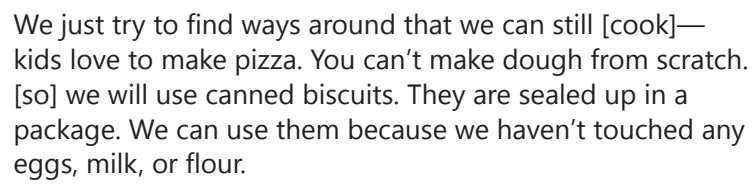
[so] we will use canned biscuits. They are sealed up in a package. We can use them because we haven't touched any eggs, milk, or flour.

Fiona suggested,

You can get some tortilla shells, pepperoni, and stuff that really don't have to be cooked, we made those and cheese. And the kids loved it. We can make wraps. It's just a matter of stepping out of the box.

Facilitator 3: Providers Perceived Mealtime Conversations as a Feasible Avenue to Deliver NE. Providers perceived the use of mealtime conversations as a feasible avenue for implementing NE compared with hands-on activities. Providers explained the use of mealtime conversations for implementing $N E$, such as allowing for frequent activities to be available to children at times outside of a formal hands-on lesson.

Abby explained,

I know we don't do it [NE] every day, but mealtime conversations can happen more frequently in our building and our classroom. I think it would be more of a challenge if we didn't eat in the classroom.

Providers reiterated the feasibility of implementing daily informal NE through mealtime conversations vs more formal hands-on activities and posters. Trisha explained,

The hands-on experiences sometimes take a lot of effort, and some of the teachers don't want to do it. And mealtime conversations are always great. I' $m$ in different classrooms, and I hear them talking. "Have you ever eaten this before? What does it taste like?" So I think that we do a lot of that. But basically, like some of the visuals, I guess, are not provided for us. 
Hannah expressed concern with hands-on sensory education and messes:

I can't have my kids touching any food. It would be a food fight everywhere. Then, clean up-we're changing clothes. It would be an uphill battle- that would be hard to do.

Maureen explained,

We are really going to focus on nutrition and pizza this week. But then next week, we might not talk about anything nutrition-wise, except maybe having conversations at lunchtime.

\section{Discussion}

The Academy benchmarks recommend that child-care providers deliver NE to young children. ${ }^{2}$ Yet, quantitative studies have reported that the provision of NE in child-care settings is limited ${ }^{14-18}$ and qualitative studies show that providers lack understanding of what qualifies as NE. ${ }^{19-21}$ Contrary to these studies, most of the providers in our study reported delivering the Academy's NE benchmarks to children and did not report a lack of understanding regarding what constitutes NE. Providers' perspectives about the Academy NE benchmarks emerged within the framework of their perceived motivators, facilitators, and barriers for delivering NE to young children. These findings reinforce the value of using a guiding framework such as the Academy benchmarks to define NE to gain a better understanding of providers' perspectives regarding delivering NE to children in their care. Nutrition and dietetics practitioners, including registered dietitian nutritionists (RDNs) and policy makers, can leverage the providers' motivators and facilitators and address their barriers to promote implementation of NE in child-care settings.

Overall, providers perceived that NE is important for children and were motivated to deliver NE because of benefits such as improving preschoolers' acceptance of new foods and knowledge regarding healthy and unhealthy foods. These providers' motivations to deliver NE in child-care settings are consistent with quantitative experimental research demonstrating the positive influence of NE interventions on children's eating behaviors. ${ }^{2,9,12,35}$ The present study providers were also motivated to deliver hand-on activities by working around policy restrictions. However, a unique study finding is that providers described implementing food-based hands-on activities (eg, home-made modeling clay, using canned biscuits for pizza) that may be limiting in some areas. These findings identify a training area for providers regarding appropriate food-based hands-on experiences for NE. The Academy's benchmarks recommend that childcare providers should receive training about child nutrition and strategies for the development of children's healthy eating habits. ${ }^{2}$ Unfortunately, providers have limited exposure to nutrition training, ${ }^{36}$ which may influence their ability to teach quality NE to children. ${ }^{21,37}$

The study providers perceived that it was easy to deliver NE because they had access to resources such as books and posters that offered repeated visual cues and did not require much planning. However, lack of monetary resources discouraged providers from practicing hands-on NE. Although HS programs receive federal funding and training, Head Start staff consistently reported lack of funding as a barrier to NE and staff professional development. ${ }^{21,38}$ Consistent with previous studies, providers in our study identified approaches for minimizing this barrier that will require provision of federal and state funds specific to $\mathrm{NE}^{21,38}$; educating child-care staff about free nutrition resources ${ }^{39}$ such as books, posters, curricula provided by the US Department of Agriculture Team Nutrition, ${ }^{40,41}$ and programs through the Cooperative Extension System. Through the Cooperative Extension System, land-grant universities provide research-based information through noneformal education and curricula to a range of audiences, including child-care providers. ${ }^{9,35,41}$ Receiving training and technical assistance and free resources such as books and activity sheets also facilitated NE in child-care settings. ${ }^{19,39,42}$

Across each primary theme, researchers observed a common trend among providers regarding the use of mealtime conversations as a feasible avenue for implementing $\mathrm{NE}$, and a potential solution for overcoming barriers related to funding and policy restrictions. Further, providers offered a novel insight for delivering NE by integrating mealtime conversations with NE concepts such as food-based sensory exploration, health benefits of foods, and modeling healthy eating. Our study findings are supported by recommendations that mealtimes can offer opportunities for learning through foods (eg, color, shape, texture, and smell) to encourage children to try new foods and eat nutritious foods. ${ }^{2,3}$ Further, quantitative work by Sigman-Grant and colleagues ${ }^{36}$ defined key mealtime practices for a supportive feeding environment. Supportive feeding environment included trying new foods as well as having conversations and teaching at mealtimes. Child-care setting mealtimes may also offer an ideal setting for integrating NE concepts with conversations because younger children are greatly influenced by adults in eating environments. ${ }^{43}$ When adults sit, eat meals with children, and enthusiastically model healthy eating it improves children's novel food acceptance and dietary intake. ${ }^{44}$ Therefore, this qualitative study drawing from the perspectives of childcare providers strengthens support to existing obesity prevention policies, ${ }^{2,3}$ and quantitative research studies ${ }^{36,45}$ recommending mealtimes as an avenue for delivering informal, daily NE in child-care settings for encouraging healthy eating in children.

While recognizing the presence of these recommendations $s^{2,3}$ and findings $\mathrm{s}^{36,45}$ related to the ease and importance of mealtime $\mathrm{NE}$, previous studies have indicated mealtime as a missed opportunity for educating children about nutrition. ${ }^{46,47}$ Observational research indicates that meaningful nutrition-related conversations between providers and children are rare and additional training in this area may be needed. ${ }^{46,48}$ Providers have reported being overwhelmed with other tasks at mealtimes, making education difficult. They state children were often distracted and unable to focus on eating. ${ }^{49}$ Providers have expressed frustration and requested resources for dealing with food refusal when fostering healthy eating in preschoolers during mealtime. ${ }^{50}$ Children's food refusal leads providers to use controlling feeding practices as a straightforward approach to encourage preschoolers to try new foods and eat fruits and vegetables. 48,50,51 Unfortunately, such practices are a risk factor for poor eating habits and childhood obesity. ${ }^{52,53}$ In addition to the aforementioned challenges to mealtime $\mathrm{NE}$, lack of availability of nutritious foods and beverages at mealtime ${ }^{54}$ and provider nutrition knowledge ${ }^{37}$ 
may attenuate the quality of NE at mealtime and fail to influence children's eating behaviors. Given our study results regarding the feasibility and importance of implementing NE through mealtime conversations, these findings highlight the complexity of the mealtime environment and identify a direction and need for research regarding examining the feasibility and effectiveness of NE at mealtime.

Some study limitations should be noted. This secondary qualitative study examined state-licensed center-based non-Hispanic white and African-American providers' perceptions in Central IIlinois to get appropriate representation from the primary quantitative study. The reduced geographic scope may have limited generalizability of the findings to other regions as well as culturally. Future studies should include a culturally diverse sample, including Hispanic and Asian providers. However, purposive sampling ${ }^{23}$ ensured that the present study providers represented a variety of backgrounds, specifically in relation to the kind of child-care program (eg, Head Start, CACFP-funded, and non(ACFP) where they cared for children, age, education, and years of experience (see the Table). Also, the 18 providers represented 14 unique child-care sites. Given that providers were interviewed regarding 18 cards each listing one benchmark, the content of the other cards may have driven providers' responses. However, all 18 cards represented different focus areas related to feeding practices, parent communication, and NE for children and each card was presented individually to a provider to follow-up her response about the individual card throughout the interview. Semistructured interviews may introduce social desirability bias where providers may have reported positive responses for practicing NE-related activities. However, the lead author assured participants that responses would be kept confidential.

\section{Conclusions}

Findings from the present study have several implications for tailoring training programs for child-care providers to harness motivators and facilitators and address barriers to the implementation of NE in child-care settings. RDNs, as well as other nutrition and dietetics practitioners, can play a primary role in addressing these implications. The role of RDNs in childcare settings has most often been limited to reviewing menus and determining whether CACFP guidelines are followed; however, our study highlights a growing scope of practice for RDNs to help child-care providers obtain and maintain foundation knowledge of Academy benchmarks and appropriate NE strategies. Providers perceived NE was important and were motivated to deliver NE to children because it encouraged children to try new and healthy foods. Leveraging these NE benefits of children's novel food acceptance and healthy eating may offer ways to motivate providers to deliver NE at mealtime. Providers perceived that mealtime conversations offer a feasible and low-cost avenue to deliver informal, daily NE in child-care settings. Providers integrated NE during mealtime conversations through food-based sensory exploration as well as communicating health benefits of foods. RDNs and nutrition educators can train providers regarding integrating mealtime conversations with NE. This approach to NE may offer a low-cost and feasible opportunity to providers to deliver daily NE to children. Future work could investigate the feasibility of incorporating the mealtime NE strategies in varying child-care contexts (Head Start, CACFP, and non-CACFP), and how it influences child-care providers' and parents' mealtime feeding practices and children's nutrition knowledge, eating behaviors, and dietary intake. Providers perceived that NE through books, posters, and hands-on activities are easier to implement when providers are offered access to and given funding for these resources. RDNs and nutrition educators can work with child-care administrators to identify resources and community partners for facilitating the implementation of these activities. Providers perceived restrictive policies as a barrier to implementing NE through hands-on cooking activities. Therefore, hands-on cooking activities may be easier to implement when policy makers, nutrition educators, and food safety professionals work together to develop standards regarding what qualifies as an appropriate food-based handson NE experiences, while also providing training on hands-on activities that are low-cost, feasible, and meet the food safety standards for state licensing. More research is warranted that reviews regulations regarding NE policies and enforcement across states and identifies how varying child-care contexts (Head Start, CACFP, and non-CACFP) and policies influence provider training and NE in child care. Further, as the Academy's NE benchmarks are updated, continued research on the adaptation and delivery using those updates is needed.

\section{References}

1. American Academy of Pediatrics, American Public Health Association, National Resource Center for Health and Safety in Childcare and Early Education. Caring for our children: National health and safety performance standards; guidelines for early care and education programs. 3rd edition. http://nrckids.org/CFOC3/index.html (Updated 2011; accessed April 6, 2012)

2. Neelon SEB, Briley ME. Position of The American Dietetic Association: Benchmarks for nutrition in childcare. J Am Diet Assoc. 2011;111(4):607-615.

3. Institute of Medicine. Early Childhood Obesity Prevention Policies. Washington, DC: The National Academies Press; 2011.

4. Larson N, Ward DS, Neelon SB, Story M. What role can child-care settings play in obesity prevention? A review of the evidence and call for research efforts. J Am Diet Assoc. 2011;111(9):1343-1362.

5. de Droog SM, Buijzen M, Valkenburg PM. Enhancing children's vegetable consumption using vegetable-promoting picture books. The impact of interactive shared reading and character-product congruence. Appetite. 2014;73:73-80.

6. Martinez LC, Gatto NM, Spruijt-Metz D, Davis JN. Design and methodology of the LA Sprouts Nutrition, Cooking and Gardening Program for Latino youth: A randomized controlled intervention. Contemp Clin Trials. 2015;42:219-227.

7. Kannan S, Smith R, Foley C, et al. FruitZotic: A sensory approach to introducing preschoolers to fresh exotic fruits at head start locations in Western Massachusetts. J Nutr Educ Behav. 2011;43(3):205-206.

8. Lisson S, Goodell LS, Dev D, Wilkerson K, Hegde AV, Stage VC. Nutrition education resources in North Carolina-based Head Start preschool programs: Administrator and teacher perceptions of availability and use. J Nutr Educ Behav. 2016;48(9):655-663.

9. Sigman-Grant $M$, Byington TA, Lindsay AR, et al. Preschoolers can distinguish between healthy and unhealthy foods: The all 4 kids study. $J$ Nutr Educ Behav. 2014;46(2):121-127. 
10. Dazeley P, Houston-Price C. Exposure to foods' non-taste sensory properties. A nursery intervention to increase children's willingness to try fruit and vegetables. Appetite. 2015;84:1-6.

11. Heath $P$, Houston-Price $C$, Kennedy OB. Let's look at leeks! Picture books increase toddlers' willingness to look at, taste and consume unfamiliar vegetables. Frontier Psychol. 2014;5(191):1-11.

12. Williams PA, Cates SC, Blitstein JL, et al. Nutrition-education program improves preschoolers' at-home diet: A group randomized trial. J Acad Nutr Diet. 2014;114(7):1001-1008.

13. Dev DA, McBride BA. Academy of Nutrition and Dietetics benchmarks for nutrition in childcare 2011: Are child-care providers across contexts meeting recommendations? J Acad Nutr Diet. 2013;113(10):1346-1353.

14. Sisson SB, Campbell JE, May KB, et al. Assessment of food, nutrition, and physical activity practices in Oklahoma child-care centers. J Acad Nutr Diet. 2012;112(8):1230-1240.

15. Nanney MS, LaRowe TL, Davey C, Frost N, Arcan C, O'Meara J. Obesity prevention in early child care settings: A bistate (Minnesota and Wisconsin) assessment of best practices, implementation difficulty, and barriers. Health Educ Behav. 2017;44(1):23-31.

16. Benjamin Neelon SE, Vaughn A, Ball SC, McWilliams C, Ward DS. Nutrition practices and mealtime environments of North Carolina childcare centers. Child Obes. 2012;8(3):216-223.

17. Martyniuk OJ, Vanderloo LM, Irwin JD, Burke SM, Tucker P. Comparing the nutrition environment and practices of home-and center-based child-care facilities. Public Health Nutr. 2016:1-10.

18. Tovar A, Risica P, Mena N, Lawson E, Ankoma A, Gans KM. An assessment of nutrition practices and attitudes in family child-care homes: Implications for policy implementation. Prev Chronic Dis. 2015;12:E88.

19. Lanigan JD. The relationship between practices and childcare providers' beliefs related to child feeding and obesity prevention. J Nutr Educ Behav. 2012;44(6):521-529.

20. Derscheid LE, Umoren J, Kim SY, Henry BW, Zittel LL. Early childhood teachers' and staff members' perceptions of nutrition and physical activity practices for preschoolers. J Res Child Educ. 2010;24(3):248-265.

21. Carraway-Stage V, Henson SR, Dipper A, Spangler H, Ash SL, Goodell LS. Understanding the state of nutrition education in the Head Start classroom: A qualitative approach. Am J Health Educ. 2014;45(1):52-62.

22. Braun V, Clarke V. Using thematic analysis in psychology. Qual Res Psychol. 2006;3(2):77-101.

23. Harris JE, Gleason PM, Sheean PM, Boushey C, Beto JA, Bruemmer B. An introduction to qualitative research for food and nutrition professionals. J Am Diet Assoc. 2009;109(1):80-90.

24. Child and Adult Care Food Program. US Department of Agriculture. http://www.fns.usda.gov/cnd/care/ProgramBasics/Meals/Meal Patterns.htm\#Child Breakfast (Updated 2012; accessed April 24, 2012)

25. Johnson SL, Ramsay S, Shultz JA, Branen LJ, Fletcher JW. Creating potential for common ground and communication between early childhood program staff and parents about young children's eating. $J$ Nutr Educ Behav. 2013;45(6):558-570.

26. Rosenthal MS, Crowley AA, Curry L. Family childcare providers' selfperceived role in obesity prevention: Working with children, parents, and external influences. J Nutr Educ Behav. 2013;45(6):595-601.

27. Price EA. Factors Influencing Feeding Styles Used by Staff During Meals with Young Children in Group Settings [doctoral thesis]. Moscow, ID: University of Idaho; 2005.

28. Dev DA, Speirs KE, McBride BA, Donovan SM, Chapman- Novakofski K. Head Start and childcare providers' motivators, barriers and facilitators to practicing family-style meal service. Early Child Res Q. 2014;29(4):649-659.
29. Rugg G, McGeorge P. The sorting techniques: A tutorial paper on card sorts, picture sorts and item sorts. Expert Systems. 1997;14(2):80-93.

30. Tong A, Sainsbury P, Craig J. Consolidated criteria for reporting qualitative research (COREQ): A 32-item checklist for interviews and focus groups. Int J Qual Health Care. 2007;19(6):349-357.

31. Krueger RA, Casey MA. Focus Groups: A Practical Guide for Applied Research. Thousand Oaks, CA: Sage Publications, Inc; 2008.

32. Bowen GA. Naturalistic inquiry and the saturation concept: A research note. Qual Res. 2008;8(1):137.

33. Hoover RS, Koerber AL. Using NVivo to answer the challenges of qualitative research in professional communication: Benefits and best practices tutorial. Prof Comm. 2011;54(1):68-82.

34. Patton MQ. Qualitative Evaluation and Research Methods. Thousand Oaks, CA: Sage Publications, Inc; 1990.

35. Farfan-Ramirez L, Diemoz L, Gong EJ, Lagura MA. Curriculum intervention in preschool children: Nutrition Matters!. J Nutr Educ Behav. 2011;43(suppl 4):S162-S165.

36. Sigman-Grant M, Christiansen E, Fernandez G, et al. Peer reviewed: Childcare provider training and a supportive feeding environment in childcare settings in 4 states, 2003. Prev Chron Dis. 2011;8(5):A113.

37. Sharma S, Dortch KS, Byrd-Williams C, et al. Nutrition-related knowledge, attitudes, and dietary behaviors among head start teachers in Texas: A cross-sectional study. J Acad Nutr Diet. 2013;113(4):558-562.

38. Hughes CC, Gooze RA, Finkelstein DM, Whitaker RC. Barriers to obesity prevention in Head Start. Health Aff. 2010;29(3):454.

39. Lyn R, Evers S, Davis J, Maalouf J, Griffin M. Barriers and supports to implementing a nutrition and physical activity intervention in childcare: Directors' perspectives. J Nutr Educ Behav. 2014;46(3):171-180.

40. Nutrition and Wellness Tips for Young Children: Provider Handbook for the Child and Adult Care Food Program. Alexandria, VA: US Depts of Agriculture and Health and Human Services; 2012.

41. Dunn C, Thomas C, Pegram L, Ward D, Schmal S. Color Me Healthy, preschoolers moving and eating healthfully. J Nutr Educ Behav. 2004;36(6):327-328.

42. Markides BR, Crixell SH, Thompson C, Biediger-Friedman L. Staff workshop improves child care center menus in south central Texas: A Best Food for Families, Infants, and Toddlers (Best Food FITS) intervention. J Nutr Educ Behav. 2017;49(5):440.e1.

43. Addessi E, Galloway AT, Visalberghi E, Birch LL. Specific social influences on the acceptance of novel foods in 2-5-year-old children. Appetite. 2005;45(3):264-271.

44. Hendy $\mathrm{H}$, Raudenbush B. Effectiveness of teacher modeling to encourage food acceptance in preschool children. Appetite. 2000;34(1):61-76.

45. Mita SC, Gray SA, Goodell LS. An explanatory framework of teachers' perceptions of a positive mealtime environment in a preschool setting. Appetite. 2015;90:37-44.

46. Gable S, Lutz S. Nutrition socialization experiences of children in the Head Start program. J Am Diet Assoc. 2001;101(5):572-577.

47. Persson Osowski C, Göranzon H, Fjellström C. Teachers' interaction with children in the school meal situation: The example of pedagogic meals in Sweden. J Nutr Educ Behav. 2013;45(5):420-427.

48. Ramsay SA, Branen LJ, Fletcher J, Price E, Johnson SL, Sigman- Grant M. "Are you done?" Childcare providers' verbal communication at mealtimes that reinforce or hinder children's internal cues of hunger and satiation. J Nutr Educ Behav. 2010;42(4):265-270.

49. Lumeng JC, Kaplan-Sanoff M, Shuman S, Kannan S. Head start teachers' perceptions of children's eating behavior and weight status in the context of food scarcity. J Nutr Educ Behav. 2008;40(4):237-243. 
50. Mita SC, Li E, Goodell LS. A qualitative investigation of teachers' information, motivation, and behavioral skills for increasing fruit and vegetable consumption in preschoolers. J Nutr Educ Behav. 2013;45(6):793-799.

51. Dev DA, McBride BA, Speirs KE, Blitch KA, Williams NA. "Great job cleaning your plate today!" Determinants of child-care providers' use of controlling feeding practices: An exploratory examination. J Acad Nutr Diet. 2016;116(11):1803-1809.

52. Dev DA, McBride BA, Fiese BH, Jones BL; on behalf of the STRONG Kids Research Team. Risk factors for overweight/obesity in preschool children: An ecological approach. Child Obes. 2013;9(5):399-408.

53. Birch LL, Fisher JO, Davison KK. Learning to overeat: Maternal use of restrictive feeding practices promotes girls' eating in the absence of hunger. Am J Clin Nutr. 2003;78(2):215.

54. Ball SC, Benjamin SE, Ward DS. Dietary intakes in North Carolina child-care centers: Are children meeting current recommendations? J Am Diet Assoc. 2008;108(4):718-721.
Potential Conflict of Interest - No potential conflict of interest was reported by the authors.

Funding/Support - This research was funded, in part, by grants from the US Department of Health and Human Services Administration of Children and Families/ Office of Planning, Research, and Evaluation (grant no. 90YR0052) and the Illinois Trans-Disciplinary Obesity Prevention Seed Grant Program.

Acknowledgments - The authors thank Karen Chapman-Novakofski, PhD, RD; Sharon Donovan, PhD, RD; and Barbara Fiese, PhD, for providing advice on the study. 


\section{Introduction}

Thank you very much for agreeing to participate in this interview. My name is Dipti A. Dev, I am a graduate student at the University of Illinois at Urbana-Champaign.

Today, I am going to interview you about your views regarding feeding guidelines ${ }^{\text {a }}$ for preschool-aged children (aged 2 to 5 years) attending child care. This study is not an assessment of whether your program is meeting certain standards, for example the Head Start or Child and Adult Care Food Program standards. We expect that most programs have not adopted many of these guidelines. This is because these guidelines are not currently an explicit part of any child-care standards. Through this study we wish to take a collaborative approach with child-care providers and bridge disconnect between policy makers and child-care staff. This interview is a chance for you to describe some of the challenges you are facing to implement these guidelines in your program.

Everything you say will be kept confidential. You will not be quoted by name. Our report on the interviews will describe the range of views expressed by staff across programs, but specific comments will not be attributed to specific individuals or programs. I also ask that you not repeat any of our discussion after you leave today.

I would like to record our interview discussion using this digital recorder so I can listen to it later, when I write up my notes. No one outside of our research team will listen to the recordings. After my notes are finalized, I will erase/destroy the recordings. If you want to say anything that you don't want recorded, please let me know and I will be glad to pause the digital recorder. Do you have any objections to my recording our discussion?

The discussion will last about an hour, and we will not take any formal breaks. But please feel free to get up at any time to stretch or use the restroom.

Once again, thank you for coming today. Do you have any questions before we get started?

Interview Sequence

Part 1. Sorting the cards

Here is a stack of cards that list guidelines for feeding children (2-5 years) in child care.

Could you put these cards into 3 piles:

1. One pile for guidelines that your center uses,

2. One for guidelines that the center doesn't use, and

3. One for guidelines that you haven't heard about or are unsure about. ${ }^{\text {b }}$

Now, could you sort the cards your center uses into another 3 piles:

1. Those that are easy to do,

2. Those that you sometimes find hard to do, and

3. One pile for really hard to do.

Part 2. Follow-up to explore provider motivators, facilitators and barriers

Let's begin with guidelines that your center uses:

a. Interviewer moves through each card in the stack of guidelines that are "easy to do."

i. What are the main reasons for doing (this)? What do you think are the most important reasons for following (this guideline)

ii. Why is (this) easy to do?

iii. What advice would you give to providers who say that they are not able to follow (this guideline)?

b. Interviewer moves through each card in the stack that are "sometimes hard to do" and then "really hard to do."

i. Why is this hard to do? / What prevents you from meeting (this guideline)?

ii. What are the main reasons for doing (this)? / What do you think are the most important reasons for following (this guideline)

iii. If you could change one thing to make (this guideline) easy to do, what would it be? What would make it easier to meet (this guideline)?

c. Let's look at this stack here. (Interviewer points to stack that aren't used.)

i. Why do you think the center doesn't use these? / What are the main reasons for the center not doing (this)? / What prevents the center from doing (this)?

(continued on next page)

Figure. Child-care provider semistructured interview protocol. 
Part 3. Conclusion

We are about done. Is there anything else you would like to add?

Do you have any questions?

${ }^{\text {a}}$ The guidelines constituted 18 benchmarks (listed on 18 different cards) including two cards for nutrition education: providers incorporate nutrition education into their daily routines with children through books, posters, hands-on experiences, and mealtime conversations; and providers help children learn about food by engaging their senses such as touching and smelling new foods is encouraged as a step toward tasting outlined in the Position Paper by the Academy of Nutrition and Dietetics benchmarks for Nutrition in Child Care.

${ }^{b}$ No providers identified a benchmark that they had not heard about or were unsure of.

Figure. (continued) Child-care provider semistructured interview protocol. 